\title{
OPTICAL CHARACTER DETECTION ON INDUSTRIAL PRODUCTS
}

\author{
Vít PASKER, Robert HLAVICA, Ondřej GRYCZ, Hana ŠPAČKOVÁ, Martin MENŠíK
}

VSB - Technical University of Ostrava, Ostrava, Czech Republic, EU, vit.pasker@vsb.cz

https://doi.org/10.37904/metal.2019.772

\begin{abstract}
The article deals with the acquisition of character positions from the input image and with modifications to increase the accuracy of recognition that precedes it. As the input image is meant a camera output or a photo of text information in the surface on different types of blanks. The input image itself contains many defects, for example noise, holes, light reflection, uneven surface caused by cutting or welding etc. All of these defects have to be eliminated to increase the accuracy of the algorithms for image recognition. In the article, there are described methods of improving the input image, which are suitable for all types of text, but it is mainly aimed at industrial products and materials that exhibit a large percentage of defects caused by metallurgical processes.
\end{abstract}

Keywords: Image recognition, image filters, blank defects, binarization, character detection.

\section{INTRODUCTION}

Computer recognition of characters and text is nowadays possible to be seen all around us. Because of its great potential, ease of work and relatively small error rates, is used more and more frequently in all possible spheres - sending postal items, transferring manually filled forms into computer form, scanning ID cards or recognizing car registrations. There are more procedures and methods to recognize the text. The main and most common feature is Optical Character Recognition (OCR). With this method, the input image is mechanically or electronically converted into machine-encoded text. The input image is the most frequently scanned document, photo, or video. Nowadays, the RFID technology is increasingly used, with an identification number label attached to the product. Special readers are then used to read the code, or the code can be decrypted by software from the image. Among the disadvantages of labels are their mechanical susceptibility to destruction and the tendency to fall off during mechanical processes. An important, practically necessary, process is the adjustment and preparation of the input image. The image itself contains many errors and imperfections that reduce the success of proper character recognition. It is possible to get quality results with high resolution recognition after proper image editing. On industrial products, these defects are even more noticeable and more widespread, so more and more sophisticated algorithms are needed to reduce these disturbing elements. [1]

\section{TECHNIQUES TO REDUCE CHARACTER READING ERROR}

The image may contain (and usually contains) certain errors, whether the input device (camera), the surface of the material, or the imperfections of the characters themselves. One of the major mistakes and possibilities of their elimination are:

- Removal of stains - this is a basic process for reducing error rates. Spots are usually caused by the dirty surface of the sensor (camera). The most effective against this error is prevention, by regularly cleaning the camera lens and general hardware scanning. Once this error is embedded in the resulting image from which the characters are to be recognized, the solution is more complex and is carried out similarly to the recognition of the characters themselves - instead of characters, the spots are recognized according to the pre-learned shapes. 
- $\quad$ Deleting tables and charts - These "bugs" are mainly contained in various forms and documents that contain a large number of frames and objects that enhance the clarity of a person when filling in them. For the text recognition algorithm, however, the existence of these elements is very poor and often leads to improper detection. Therefore, it is necessary to remove the table and the various lines (which are clearly detected as lines, not as part of the letters) before the text is recognized.

- $\quad$ Edge removal - Ideally, the camera is turned on the product so that the edges are as small as possible. However, this is often not possible, so the edges are erased by software. Existence of the margins does not have to mean a malfunction of the algorithm that detects the characters, but greatly simplifies the work and reduces the number of cycles the algorithm has to manage. At the same time, this change in the size of the surveyed pattern will positively affect other processes that will run much faster.

- Color adjustment - The text recognition algorithm works based on an image input with a unique color spectrum that can be logically converted into two states - the pixel is the text or the pixel is the background. Of course, this is a very simple formula that applies only to machine-typed texts on a perfectly pure background. The main color treatment is so called binarization. In this process, each individual image pixel is converted to logic 1 or logic 0 according to defined decision levels (for colors, these are practically three different decision levels for the RGB spectrum). The output of binarization is then a black and white image that can be presented as input to the text recognition algorithm.

- Alignment of the font tilt - practically can be defended against this error in two ways. The first is to build an algorithm that will be used to straighten the characters. Such a method may be more demanding to implement, and it can irritate the characters irreversibly so that they cannot be recognized. A simpler and more efficient method is to increase the training set and incorporate characters with different slopes. Detection will take place just as with straight text. [2,3]

The input image editing process for the character recognition algorithm is shown in the Figure 1.

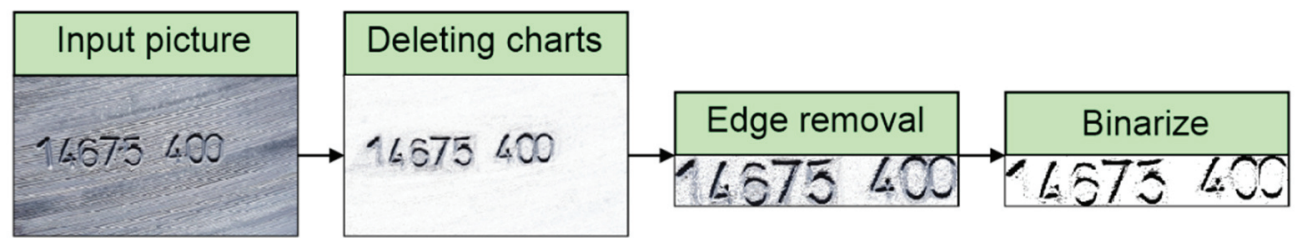

Figure 1 Process of editing the input image

\section{ALGORITHM OF OPTICAL DETECTION OF CHARACTER POSITIONS}

In the MATLAB programming environment, a sequence of algorithms was created to take care of the individual input image editing processes. The result of this sequence is information about the layout of characters in the image - their number, coordinates, and size. Individual characters will be used to recognize the entire text. There are also OCR types that are able to compare whole words. This solution may be more effective for short words, with longer words it may be a problem to find a suitable pattern. It is also more complicated to treat the slope of individual letters (some are tilted, others are not). Whole word comparisons can be used where input is expected, for example when choosing from predefined options. In this case, the recognition speed is much lower than with sequential decoding of characters.

First, it is necessary to change the size of the input image. In many cases, the image is too large due to the high resolution, so it has to be reduced to greatly speed up the whole process of input adjustment and its subsequent recognition. To resize an image, MATLAB has a simple function, but since it is the purpose of creating atomic algorithms that are transferable between different programming languages, it is necessary to define and create a custom function for this purpose. The algorithm works by dividing the image into $5 \times 5 \mathrm{px}$ 
matrices. From each matrix, it calculates the amount of color contrast that is the input to a new, reduced image where it represents $1 \mathrm{px}$. The principle of the process can be seen in the Figure 2. [3]

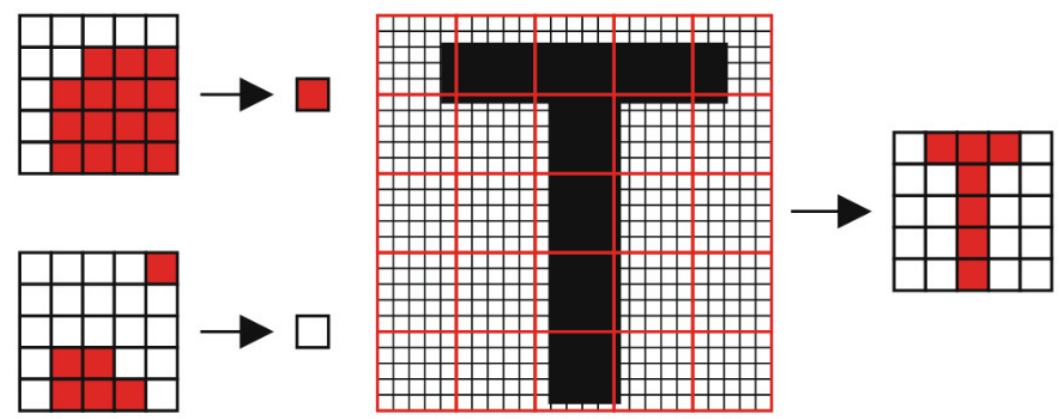

Figure 2 The principle of resizing image process

This reduced image is ready for the next processes, which are to reduce the reading error. Here, stains and lines are removed. The principles of this process are more, often used is the so-called mask. Mask is an image that contains a shape (line, circle, polygon) that is compared to the input image. It overlaps both images and removes the matching parts. An example of output after using the line elimination mask is in the Figure 3. This adjustment will greatly enhance the success of character position detection and its later recognition. For demonstration, the algorithm output from both the original image and the modified image is displayed in all procedures. [4]
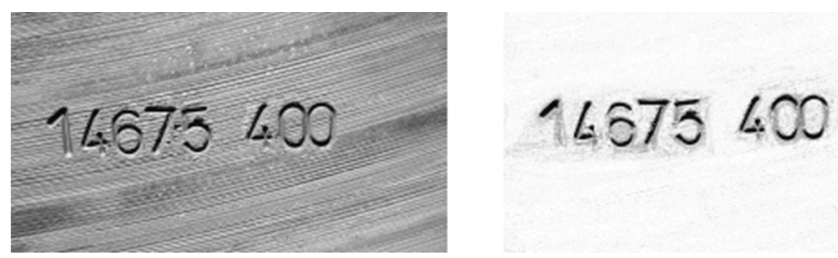

Figure 3 Input images (Left - raw input image, right - edited input image)

After mask application, the image should be trimmed by the edges. In this case, however, edge trimmer step is omitted for better view of the character position detection algorithm. The next step is to binarize the image. In the case of a good background (surface), a simple binarization is sufficient where each pixel is compared to a certain level that indicates whether the output pixel is 1 or 0 . However, for images with a poor and noised background, the evaluation would be too poor to work with. Therefore, it was created more algorithms that perform other types of binarization. The outputs of all these algorithms are evaluated and one binarized output image is created to work with. The output binarizations for the input and the edited picture are in the Figure 4. [4]
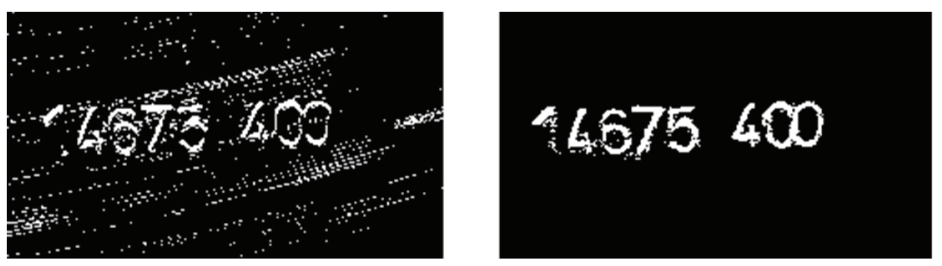

Figure 4 Binarized images (Left - raw input image, right - edited input image)

From a binary image, the position of characters can be detected. The sum of the pixels in the $\mathrm{X}$ and $\mathrm{Y}$ axes is performed in the algorithm. This sum is then compared to the decision level that determines whether the axis is a noise or a character, ie whether the information is to be discarded or considered to be the result. 
The $\mathrm{X}$-axis anomaly histogram is displayed in the Figure $\mathbf{5}$, in the Figure 6 there is a histogram of the Y-axis anomaly.
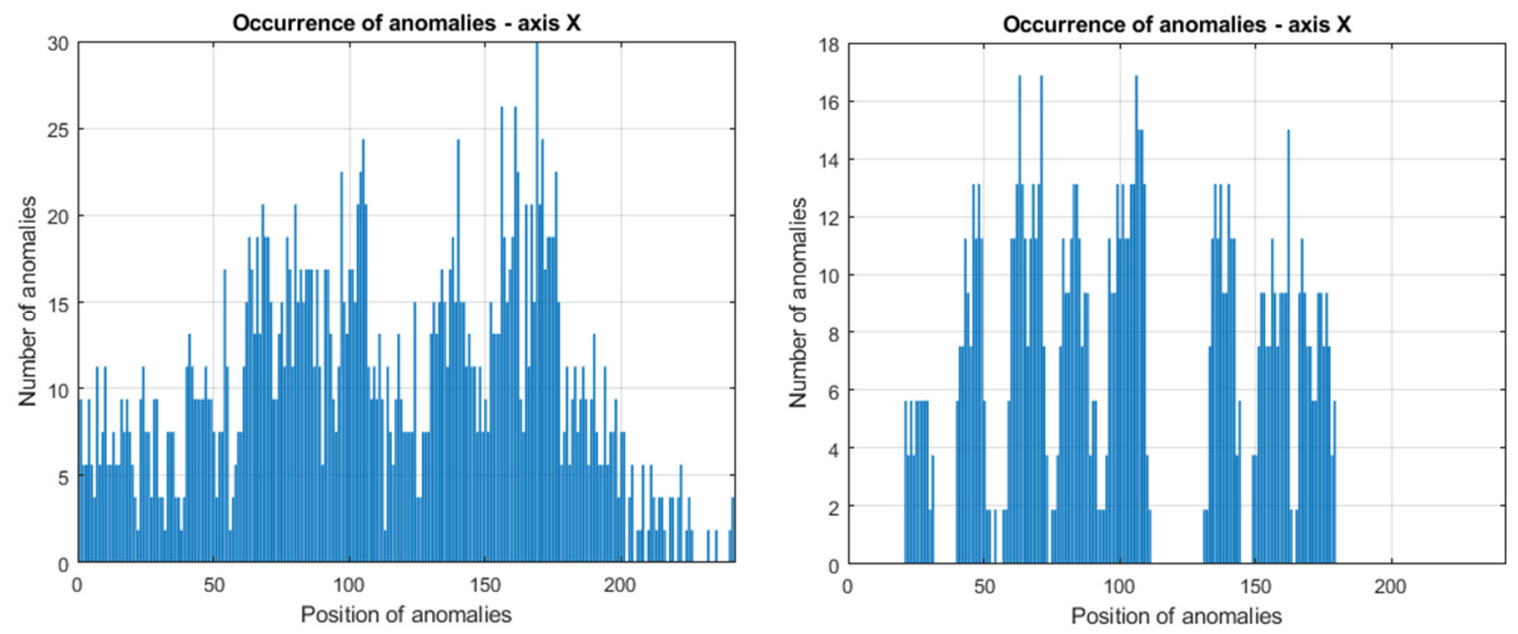

Figure 5 Occurrence of anomalies on axis X (Left - raw input image, right - edited input image)
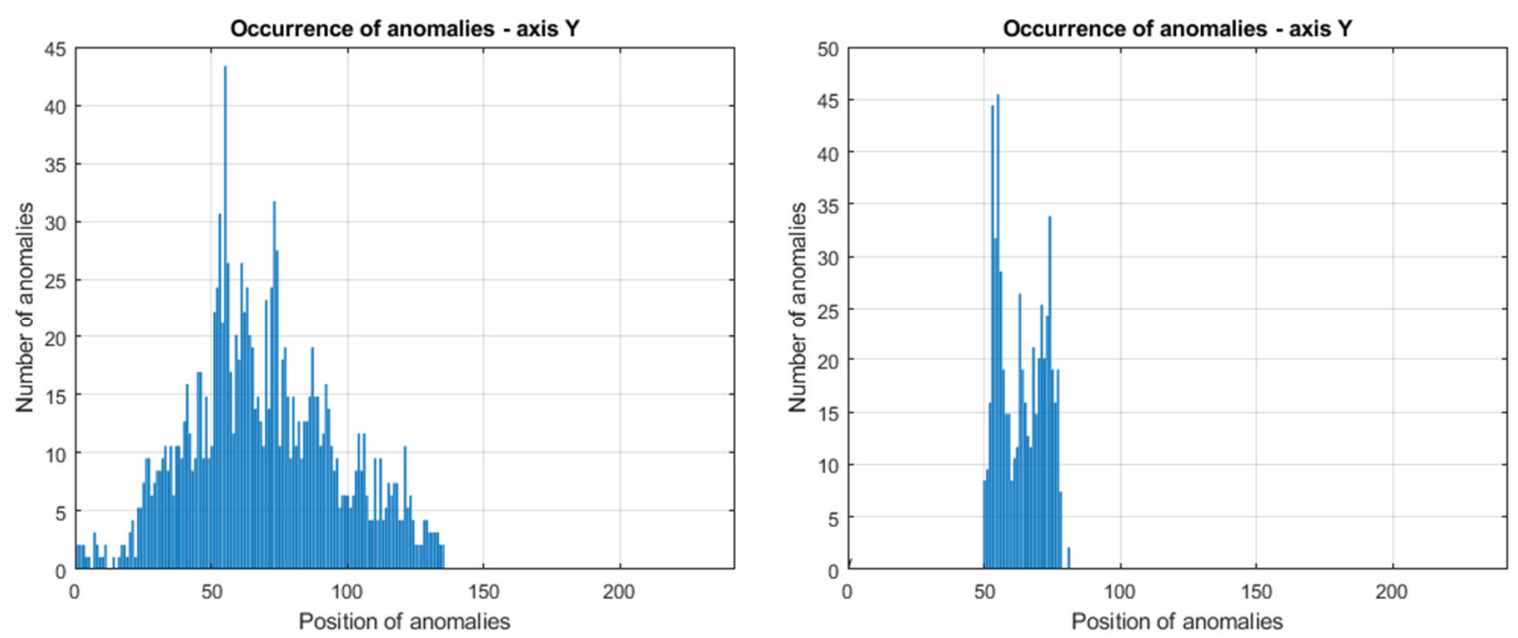

Figure 6 Occurrence of anomalies on axis Y (Left - raw input image, right - edited input image)

By linking information about the sums of pixels in each $X$ and $Y$ axis, information can be rendered in the image. Line overlay on $X$ and $Y$ lines limits the occurrence of characters. As can be seen in the Figure 7, the unadjusted input image evaluates the position of characters with very low accuracy. This output can hardly be used as input for the character recognition algorithm. On the other hand, a modified image, which has removed typical features that reduce its quality, shows great accuracy in recognizing character positions.
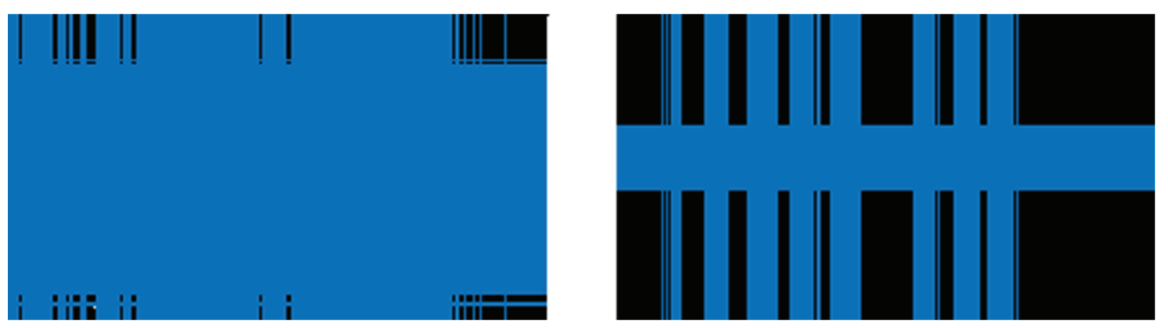

Figure 7 Output images (Left - raw input image, right - edited input image) 


\section{CONCLUSION}

Several methods and algorithms exist for detecting character position in the image. However, for most of the existing methods, the input image must have optimal properties, such as clear text on a clean background. Such algorithms are unusable when reading information from industrial materials and products that contain many imperfections, such as unsymmetrical embossed text into products or a bad surface. Because of these imperfections, algorithms have been created to remove excess lines, stains, or spreadsheets from the image. In the photo of a serial number from an industrial product with a large number of surface defects, it has been found that the untreated image has a very poor recognition of character positions. On the modified image, the algorithm was able to recognize character positions with high precision.

Improved accuracy can be achieved by a larger number of input image filters that remove unwanted objects from the image. But be careful not to eliminate parts of the actual characters, which would lead to deterioration of input data for the character recognition algorithm.

\section{ACKNOWLEDGEMENTS}

The work was supported by the specific university research of Ministry of Education, Youth and Sports of the Czech Republic No. SP2019/17 and SP2019/62.

\section{REFERENCES}

[1] DAVID, J., FRISCHER, R., STRANAVOVA, M. Usage of rfid wireless identification technology to support decission making in steel works. In METAL 2012: 21st International Conference on Metallurgy and Materials. Ostrava: TANGER, 2012, pp. 1734-1738.

[2] FRISCHER, R., DAVID, J., SVEC, P., KREJCAR, O. Usage of analytical diagnostics when evaluating functional surface material defects. Metalurgija. 2015, vol. 54, no. 4, pp. 667-670.

[3] THEODORIDIS, S., KOUTROUMBAS, K. Pattern Recognition. 2nd ed. Academic Press, 2008. pp. $163-181$.

[4] RICE, S.V., NAGY, G., NARTKER, T.A. Optical Character Recognition: An Illustrated Guide to the Frontier. Proceedings of SPIE - The International Society for Optical Engineering [online]. 1999. vol. 3967, pp. 58-69 [viewed 2019-01-15]. Available from: DOI: 10.1117/12.373511. 